\title{
MANNED SPACE EXPLORATION AND THE POSSIBILITY OF HYPOXIA*
}

\author{
R. H. LOWRY, M.D. $†$
}

THE IMMINENCE of manned space flight permeates the thinking of the world today. The flight of manned satellites is no longer a fantastic dream of the distant future, for the impending orbiting of such vehicles will herald man's first faltering footsteps into the unknown vast depths of space.

Engineering technology, combining the/specialties of aerodynamics, structures, propulsion, communications, and many other facets, has proved the feasibility of vehicular space travel. The critical design ol such vehicles demands the detailed evaluation of engineering systems for reliability, ease of maintenance. and general performance under the stress of space flight. Moreover, practical engineering design, manufacture, and test must be backed up by many man years of fundamental research in various fields such as chemistry, metallurgy, and geophysics. The rapid expansion of engineering technology has provided theoretical knowledge and facts so that men are capable of producing the vehicles which are propelled out of this world to the abyss of space. The early: unmanned vehicles have beèn fabricated and flown, but we must remember that today we are interested in manned space flight and exploration. Such an interest imposes many detailed problems which must be surmounted in our planned program for space flight. Unfortunately, our medical knowledge of this strange material called man is somewhat lacking in many facets so important in space flight. The engineer is dealing with materials and structures which have a specific yield point at some given stress. However, the aerospace medical group is responsible for the well-being of man in flight. Man, however. is a mixture of organic and inorganic substances with a mental component which decrees a rather wide variation in the ability of the various individuals to cope with combined stresses. This interphase of medicine and engineering presents a fertile area to apply the general biological sciences for the optimum integration and most efficient use of humans in the manned machine concepi associated with this bold venture into space.

The very basis of space medicine, of course, is sound clinical medicine. However, when we apply this field of medicine to man in flight, we rapidl! become involved in such problems as protective clothing against hostile enlvironments, in-flight feeding, sealed cabins with associated problems of gaseous exchange, waste disposal, toxicity, and so on. Furthermore, the selection of

*Presented at the annual meeting of the Western Divisions, Canadian Anaesthetists' Soriet!, Victoria, B.C., April 28-30, 1960

†Chief, Space Medicine Section, Aero-Space Division of Boeıng Airplane Company, Seattle, Washington, USA 
crews, including their physiological training, their survival training, the associated problems of corpuscular and electromagnetic radiation, weightlessness, remoteness, the possibility of failure of the cabin pressurization system resulting in a rapid decompression, and many other problems requires continual surveillance of the medical aspects associated with such manned flight.

An essential aspect of man's environment, whether he is on the ground or in a vehicle in flight, is his oxygen requirements. A momentary review of our basic sciences will remind us that the essential aspect of oxygen concentration as far as man is concerned is the partial pressure of the gas which reaches the alveoli. Likewise, we will remember that as the altitude increases, the partial pressure of the oxygen decreases, even though the percentage by volume of the oxygen in the environment remains constant up to many thousand feet. It is this decreasing partial pressure of oxygen as the altitude increases that causes the respiratory difficulty to our flyer. A moment's futher reflection will remind us of the oxygen dissociation curves for human blood. Oxygen combines reversibly with haemoglobin in a unique manner to form oxyhaemoglobin. Three points are paramount regarding this curve. (1) The combination of haemoglobin with oxygen is influenced by the partial pressure of the oxygen in the surrounding medium. This, of course, has a direct effect on the ability of the blood to transport oxygen to the tissues of the body at various altitudes. (2) Haemoglobin has a relatively high affinity for oxygen at certain high partial oressures of oxygen, and a relatively low affinity for oxygen at lower pressures. This, of course, forms the S-shaped curve with which you are all familiar in the xxygen dissociation graph. The practical application of such a curve is that blood has a high capacity for oxygen at the partial pressure of oxygen in the lungs when the oxygen partial pressure is relatively high, and a low capacity at the lower partial pressure of oxygen which is found in the tissues. This results in a rapid loading of oxygen in the lungs and a rapid unloading of oxygen in the tissues. (3) The third point to remember from the dissociation curve is the shift of the curve to the right or to the left, depending on the variation of the $\mathrm{pH}$ of the blood: Therefore, as the $\mathrm{pH}$ of the blood becomes more alkaline, the oxygen capacity of the haemoglobin is increased; and as the blood becomes more acidic, the percentage of oxygen saturation of the haemoglobin is decreased. As the human ascends, an appreciable hypoxia associated with approximately 84 per cent oxyhaemoglobin saturation occurs the average, at about 13,000 feet while breathing normal air. A serious handicap is noticeable around 17,000 feet, with imminent collapse, on the average, at approximately 19,000-20,000 feet. When breathing pure oxygen, however, the altitudes are increased, so that utilizing pressure breathing the appreciable hypoxia is first noted around 42,000 feet, with serious hamdicap and probably imminent collapse occurring around 44,000 or 45,000 fet altitude.

Whên discussing space flight, we must remember that for all intents and purposes our,vehicle is operating in a vacuum. It is most essential, therefore, that the astronaut be protected by being enclosed in an environment that is capable of sustaining life and also permitting hiph to efficiently carry out his 
expected work load. Protection for man in space flight will, therefore, recuuire what is known as a sealed cabin. This sealed cabin must maintain an artificial atmosphere closely monitored with rigid parameters.

Let us examine some of the primary requirements of the human body that must be considered when designing the sealed cabin of a manned space vehicle. To maintain man's mental acuity, a partial pressure of oxygen approximately equal to that found at sea level, or $160 \mathrm{~mm}$. $\mathrm{Hg}$, will be required. The internal cabin pressure should be maintained below 18,000-20,000 feet, or an equivalent of $350 \mathrm{~mm} ; \mathrm{Hg}$, in order to insure that the ambient pressure will prevent bends, chokes, and so on from developing in the astronaut during flight. The difference between the partial pressure of oxygen in the cabin and the total cabin partial pressure must be made up with nitrogen or some other inert gas in order to insure that there is no oxygen toxicity, respiratory pathology, or problem of flammability with which the astronaut will have to cope. Oxygen 'toxicity develops when the human is subjected to a partial pressure of oxygen of 425 $\mathrm{mm}$. or more for a moderate length of time. The fact that tolerance to oxygen is limited only if its partial pressure exceeds about $425 \mathrm{~mm}$. $\mathrm{Hg}$ would indicate that pure oxygen can be breathed with impunity at all altitudes above 15,000 feet where the partial pressure of the environment equals around $425 \mathrm{~mm}$. $\mathrm{Hg}$ : Herein, however, a man may encounter further difficulties if a blockage of one of the alveolar ducts of the lung occurs owing to a mucous plug or some other obstruction developing. If an astronaut is breathing pure oxygen, he would, therefore, have partial pressure resulting from only a mixture of oxygen, carbon dioxide, and water vapour in his alveolar sacs without the partial pressure of an inert gas such as nitrogen. There is then the possibility of having the oxygen absorbed through the alveolar walls with the resultant collapse of part of the lung due to the lack of partial pressure from an inert gas, such as nitrogen, remaining in the lungs and keeping them inflated.

A most important point in the operation of a sealed cabin is' the adequate removal of carbon dioxide and other toxic gases from the recycled air. Carbon diuxide in a sealed cabin environment is harmful to a man when contiruously present in excess of 3 per cent of the total atmospheric gas, and. preferably should be maintained below a concentration of 0.5 per cent. The levels of loxygen and carbon dioxide in the environment, therefore, must be adequately monitored at all times in order to maintain man's efficiency. It must also be remembered that organic and inorganic contaminants may be given off in the form of gases or vapours from the use of paints, resins, refrigerants, carbon monoxide due to smoking, human wastes, and so on, in such a sealed cabin. Such toxic compounds, even though in minute quantities, must be meticulously removed from the breathed environment during the recycling process in order to insure that acute or even chronic toxic effects will not hamper the operation of the central nervous system of the astronaut.

In addition to supplying an adequate gaseous environment in the cabin, the temperature and humidity must be controlled for the comfort and, indeed, the very existence of the human organism. While one could state many cases where hypoxia had been the dominant factor: in an emergency during terrestrial 
flight, it must be pointed out that the humidity of the atmosphere is also of utmost importance in the sealed cabin environment in order to control the fluid balance and the temperature of the body. A change in the fluid balance of the body can readily affect the acid-base ballance of the blood, therefore directly affecting the pulmonary ventilation with possibfte serfous physiological effects on the space crewman.

For short space flights, where weight is not an insurmountable obstacle, the utilization of cryogenic systems will permit a balanced atmosphere to be maintained in the cabin in an open-ended system. However, for longer flights of several weeks duration, chemical-oxygen regenefation systems utilizing hydrogen peroxide or potassium superoxide will be required to produce sufficient oxygen while maintaining a satisfactory $\mathrm{O}_{2}$ production weight ratio for efficient operation. $\mathrm{CO}_{2}$ and other toxin $\$$ will be absorbed chemically or regenerated to $\mathrm{O}_{2}$. Travel involving several months or more duration in space vehicles will require photosynthetic gas exchangers involving combination of algae and broad leaf plants for the conversion of $\mathrm{CO}_{2}$ to $\mathrm{O}_{2}$ and food for use in flight. The regeneration of water in such systems will be required for space flight operations of approximately three weeks or more, whereas the regeneration of food becomes an efficient operation in flights involving approximately three months duration or more.

The reliability of present-day sealed cabins sugglests the requirement for the astronaut to wear a full pressure suit for physidlogical protection in the event of the loss of cabin pressure in a rapid decomptession in the cabin. The wearing of such safety equipment brings forth other problems even when it is not inflated, for the garment is heavy and warm and interferes to some extent with the comfort, mobility, and the normal evaporation of perspiration and, hence, the cooling of the man. It is essential, therefore, that adequate ventilation be maintained at all times through the suit to maintain the normal body temperature of the crewman in such an environment.

Space exploration in its true sense will require the landing of vehicles on other planets. After a landing, the aircrew will leave the protective environment of their sealed cabins and explore the surrounding planets. In accordance with our best knowledge at the present time, it will be necessary for such explorers to wear constantly adequate full pressure suit clothing which not only gives a protective ambient pressure around the body, but on leaving the spacecraft must include such things $\mathrm{as}^{5}$ radiation protection, communication, and mobile environmental recycling apparatus to absorb $\mathrm{CO}_{2}$ and other toxic gases and provide fresh oxygen continually for man's use. In such instances hypoxia or lack of oxygen could result from yet another aspect, namely, the effect of radiation on any bottled gas being carried in the mobile pack; for pressurized oxygen subjected to intense radiation has the possibility of producing ozone which, of course, is highly toxic to humans.

Hypoxia, or lack of adequate oxygenation, has been discussed in its broadest aspect as a problem in the space cabin environment arising as a result of many factors that might occur during flight, rather in detail on the technical aspects of pure hypoxia due to oxygen lack per se. Associated problems of manned space 
$7 \pm$

CANADIAN ANAESTHETISTS' SOCLETY JOLRNAL

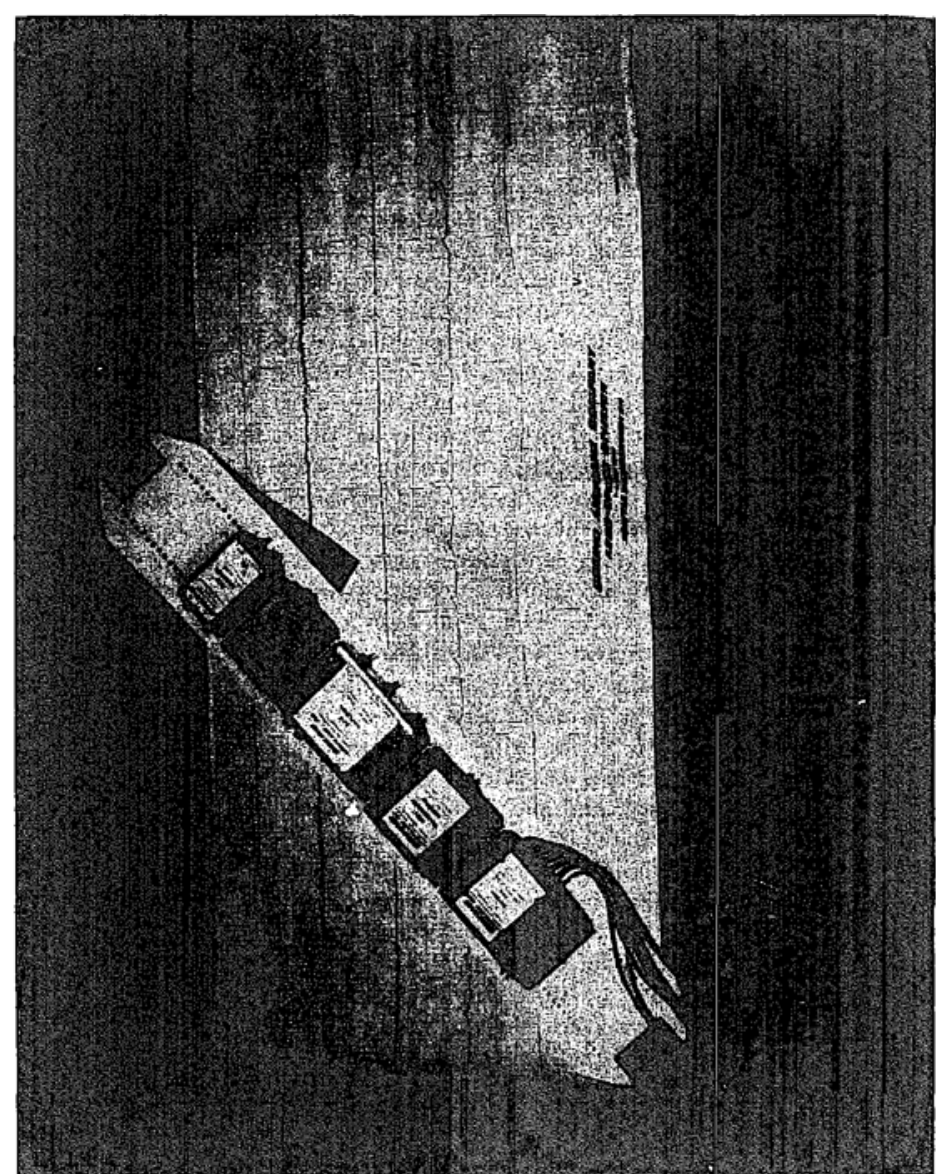

FIGURE I. Miniature physiological recording instruments developed by the Boeing Airplane Co., producing the five channel simultaneous recording of EEG, ECG, respiration, body temperature, and phonocardingraph.

flight involving combinations of heat, acceleration, vibration, and ot her physical

parameters may lead to a resultant $\mathrm{O}$ lack which has similar physiological

effects to simple hypoxia. Such problems are being cvaluated at this time. Many facets are associated with the prohlem of maintaining an appropriate 
environment to sustain man during space flight such as adequate humidity., carbon dioxide content, temperature, control of cabin toxicities, odours, etc. These facets play an equally important factor with the simple oxygen partial pressure of the sealed cabin or pressure suit for adequate insurance against hypoxia.

We should mention yet another aspect associated with this problem of hypoxia in space flight. Miniaturized electronic instrumentation is a most important aspect of both the cabin hardware and the physiological well being of the man. We could discuss the instrumentation in two main areas; namely, the cabin sensors and the physiological instrumentation. The telemetred data from both areas will be combined at the flight controller's desk giving essential information regarding the physical and physiological status during the flight. Cabin sensors refer to the miniature indicators which continually monitor the partial pressure of oxygen; the partial pressure of carbon dioxide, the temperature, the ambient pressure, vibration, and so on; and as the sophistication of the vehicle increases, these will monitor the toxicities present in the cabin environment through the use of a miniaturized mass spectograph. The second area, miniaturized physiological instruments, continually monitor the astronaut during all phases of the flight. The principal instruments are the respirometer; the electrocardiograph, the body temperature recorder, the automatic blood pressure recorder, the phonocardiograph, and the electroencephalograph.

The ageless problem of hypoxia will be an area of prime importance to the astronaut during space exploration. The combined engineering and biological aspects of the environment surrounding the man will require constant monitoring to insure that man is adequately protected and sustained in order to efficiently carry out his duties during space flight. 